\title{
Phase Matched Third Harmonic Generation of a Gaussian Laser Pulse in High-Density Quantum Plasma
}

\author{
Nisha Singh Rathore, Punit Kumar* \\ Department of Physics, University of Lucknow, Lucknow, India \\ Email address: \\ punitkumar@hotmail.com (P. Kumar) \\ ${ }^{*}$ Corresponding author \\ To cite this article: \\ Nisha Singh Rathore and Punit Kumar. Phase Matched Third Harmonic Generation of a Gaussian Laser Pulse in High-Density Quantum \\ Plasma. American Journal of Modern Physics. Vol. 5, No. 5, 2016, pp. 154-161. doi: 10.11648/j.ajmp.20160505.16
}

Received: July 22, 2016; Accepted: August 10, 2016; Published: October 6, 2016

\begin{abstract}
Third harmonic generation due to linearly polarized Gaussian laser pulse propagating through quantum plasma immersed in transverse wiggler magnetic field is studied using the quantum hydrodynamic (QHD)model. The effects associated with the Fermi pressure, the Bohm potential and the electron spin have been taken into account.Wiggler magnetic field plays both a dynamic role in producing the harmonic current and a kinematical role in ensuring phase-matching. It is shown that the harmonic radiation attain the maximum valueat an instant when the phase matching is satisfied and thereafter decreases at later duration of laser pulse. The quantum effects also add to harmonic generation in the phase-matched case.
\end{abstract}

Keywords: Quantum Plasma, Harmonic Generation, Phase Matching

\section{Introduction}

The interaction of intense laser pulse with plasma leading to harmonic generation has been an area of interest for the last thirty years. The physical phenomenon of interaction of a high intensity laser radiation with plasma leads to a number of relativistic and nonlinear effects such as self modulation, self-focusing, Raman scattering, and harmonic generation[12]. Harmonic generation of electromagnetic radiation in laser-produced plasmas and laboratory plasmas is an important nonlinear process with considerable potential for plasma diagnostics [3-12]. Although a number of high-order harmonic generations $[13,2]$ have been analyzed but the third harmonic generation $[14,15]$ has its unique place in the research related to laser plasma interactions. From last few years, a great deal of research has been focused on second and third harmonic in laser produced plasma [16-22]. Theory for coherent emission in the direction of propagation of laser beam, referred to as relativistic harmonic generation, has been derived $[1,2]$. It indicates that because of the mismatch between the phase velocities of the laser pulse and the generated harmonics and because of the collective response of the plasma, the conversion efficiency should be low unless a means for phase-matching [23] is implemented.
Experimentally, Liu et al. [24] tried to measure the third harmonic light produced from relativistic harmonic generation but ultimately could give only an upper limit on the conversion efficiency. Averchi et. al. [25] proposed a different approach to obtain phase-matched generation of high order harmonic based on the use of pulsed Bessel beams. Sheinfux et. al. [26] demonstrated a scheme for creation of periodic plasma structures by ablating a lithographic pattern for quasiphase matched harmonic generation. Shibu and Tripathi [27] have studied phasematched third harmonic generation of a laser beam propagating through a plasma channel and showed that the presence of a background density perturbation can account for phase-matching. Salih et al. [28] have studied the secondharmonic generation of a Gaussian laser beam in a self created magnetized plasma channel. Rax et al. [29] demonstrated that when an intense plane polarized laser pulse interacts with a plasma, the relativistic nonlinearities induce third harmonic radiation. Sapaev et. al. [30] demonstrated a novel method of quasiphase matching third harmonic generation in noble gases employing ultrasound. Kant et al. [31] observed the resonant third-harmonic generation of a short pulse laser from electron hole plasmas in the presence of wiggler magnetic field. It was observed that for a specific wiggler wave number value, the phase matching conditions 
for the process are satisfied, leading to resonant enhancement in energy conversion efficiency.

All the above work has been done for classical plasma. Classical plasma physics has mainly focused on regimes of high temperatures and low densities, in which quantum mechanical effects play no role. Plasma where the density is quite high and the de-Broglie thermal wavelength associated with the charged particle i.e., $\lambda_{B}=\hbar /\left(2 \pi m k_{B} T\right)$ approaches the electron Fermi wavelength $\lambda_{F e}$ and exceeds the electron Debye radius $\lambda_{D e}$ (viz., $\lambda_{B} \sim \lambda_{F e}>\lambda_{D e}$ ), the study of quantum plasma becomes important. Furthermore, the quantum effects associated with the strong density correlation start playing a significant role when $\lambda_{B}$ is of the same order or larger than the average inter-particle distance $\sim n_{o}{ }^{-1 / 3}$, i.e., $n_{0} \lambda_{B}^{3} \geq 1$ hold in degenerate plasma. However, the other condition for degeneracy is that the Fermi temperture $\left(T_{F}\right)$ which is related to the equilibrium density $\left(n_{o}\right)$ of the charged particles must be greater than the thermal temperture $(T)$ of the system [32,33]. The high-density, low-temperature quantum Fermi plasma is significantly different from the low-density, high-temperature "classical plasma" obeying Maxwell-Boltzman distribution. During the last decade, there have been many papers devoted to influence of spin on dynamics of plasma [34, 35]. Recently, the quantum kinetic studying of the waves in plasma made [36]. The growing interest in investigating new aspects of dense quantum plasmas motivated by its potential applications in modern technology [37] e.g., microelectronics devices, quantum plasma echoes, metallic nanostructures, metal clusters, thin metal films, quantum well, quantum dots, nano-plasmonic devices, quantum x-ray free electron lasers, in super dense astrophysical environment (e.g. in the interior of Jupiter, white dwarfs, and neutron stars), in high intensity laser produced plasmas,in metallic nanostructures, in nonlinear quantum optics, in dusty plasmas and in next generation of laser based plasma compression experiment (LBPC), etc.

In this paper, we study the third harmonic generation of a Gaussian laser pulse in quantum plasma in the presence of a transverse wiggler magnetic field incorporating the quantum effects including the spin-1/2 effect. We have developed the mathematical formulation with the help of the quantum hydrodynamicl (QHD) model [38, 39]. The QHD model consists of a set of equations describing the transport of charge density, momentum (including the Bohm potential) and energy in a charged particle system interacting through a self consistent electrostatic potential. QHD model is a macroscopic model and application is limited to those systems that are large compared to Fermi length of the species in the system. The advantages of the QHD model over kinetic descriptions are its numerical efficiency, the direct use of the macroscopic variables of interest such as momentum and energy and the easy way the boundary conditions are implemented.

Since quantum plasma is a highly dispersive medium, the phase matching conditions are not satisfied, thereby making the process non-resonant. If the process is made resonant, then the efficiency of the process can be enhanced significantly. Our focus is to enhance the third harmonic generation in quantum plasma by satisfying the phase matching condition in the presence of wiggler magnetic field. The wiggler provides additional momentum to make process resonant, which leads to enhance the efficiency of harmonic generation. In the process of third harmonic generation the oscillatory velocity of the plasma electrons due to pump laser field at $\left(\omega_{o}, \vec{k}_{o}\right)$ beats with a laser magnetic field to produce a second harmonic ponderomotive force at $\left(2 \omega_{o}, 2 \vec{k}_{o}\right)$. The oscillatory velocity due to this ponderomotive force betas with wiggler magnetic field $\left(0, \vec{k}_{w}\right)$ to exert a ponderomotive force at $\left(2 \omega_{o}, 2 k_{o}+\vec{k}_{w}\right)$ couples with the electron density oscillations at $\left(\omega_{o}, \vec{k}_{o}\right)$ to produce a nonlinear current at $\left(3 \omega_{o}, 3 k_{o}+\vec{k}_{w}\right)$, which drives the third- harmonic radiation.

\section{Source Currents}

Consider the propagation of a Gaussian laser pulse through quantum plasma of electron density $n_{o}$. The electric and magnetic fields of the laser pulse are,

$$
\begin{gathered}
\vec{E}_{y}=\hat{y} E_{o} \exp \left(k_{o} z-\omega_{o} t\right) \\
\vec{B}_{x}=\hat{x} \frac{c k_{o}}{\omega_{o}} E_{o} \exp \left(k_{o} z-\omega_{o} t\right) . \\
E_{o}^{2}=E_{o o}^{2} \exp \left(-\left(t-z / v_{g}\right)\right)^{2} / \tau^{2} .
\end{gathered}
$$

where $k_{o}=(\omega / c) \eta_{o}$ is the propagation constant, $\eta_{o}$ is the refractive index of the plasma and $v_{g} \approx c \eta_{o} \approx c$ is the group velocity. An external wiggler magneticfield $\vec{B}_{w}=\hat{y} B_{o w} \exp \left(k_{w} z\right)$ is applied to the plasma. The interaction dynamics is governed by the following set of QHD equations.

$$
\begin{gathered}
\frac{\partial(\gamma \vec{v})}{\partial t}=-\frac{e}{m \gamma}\left[\vec{E}+\frac{1}{c}(\vec{v} \times \vec{B})\right]-\frac{v_{F}^{2}}{n_{o}^{2}} \frac{\vec{\nabla} n^{3}}{n} \\
+\frac{\hbar^{2}}{2 m^{2} \gamma^{2}} \vec{\nabla}\left(\frac{1}{\sqrt{n}} \vec{\nabla}^{2} \sqrt{n}\right)-\frac{2 \mu_{B}}{m \hbar} \vec{S} \cdot \nabla \vec{B}, \\
\left(\frac{\partial}{\partial t}+\gamma \vec{v} \cdot \vec{\nabla}\right) \vec{S}=\left(\frac{2 \mu_{B}}{\hbar}\right)(\vec{B} \times \vec{S}), \\
\frac{\partial \gamma n}{\partial t}+\vec{\nabla} \cdot(\gamma n \vec{v})=0 .
\end{gathered}
$$

where, $\vec{v}$ is the velocity, $\hbar$ is the Planck's constant divided by $2 \pi, v_{F}$ is the Fermi velocity and $\vec{S}$ is the spin angular momentum with $\left|S_{o}\right|=\hbar / 2$ and $\mu=(-g / 2) \mu_{B}$,with $g=2.0023193$ and $\mu_{B}=e \hbar / 2 m c$ being the Bohr magneton, 
the plasma frequency and the frequency of gyration are being defined as $\omega_{p}=\left[n_{o} e^{2} /\left(m \varepsilon_{o}\right)\right]^{1 / 2} \quad$ and $\left(\omega_{o w}=e B_{o w} / m . c\right)$ respectively, where $\mathrm{e}$ and $\mathrm{m}$ are electronic charge and rest mass respectively and $\gamma$ is relativistic factor. The third term on the right-hand side of eq. (1) denotes the Fermi electron pressure. The fourth term is the quantum Bohm force and is due to the quantum corrections in the density fluctuation. The last term is the spin contribution to the momentum. The above equations are applicable even when different spin states are well represented by a macroscopic average. The wave equation for the current source is.

$$
\left(\vec{\nabla}^{2}-\frac{1}{c^{2}} \frac{\partial^{2}}{\partial t^{2}}\right) \vec{E}(\vec{r}, t)=\frac{4 \pi}{c^{2}} \frac{\partial \vec{J}}{\partial t}
$$

where, $\vec{J}$ is the current density.

From the equation of motion (eq. (2)), the components of quiver velocity at $\left(\omega_{o}, k_{o}\right)$ imparted to the plasma electron are obtained as

$$
\begin{gathered}
\vec{v}_{x}^{(1)}=v_{1 x}^{(1)} E_{o} \exp i\left(k_{o} z-\omega_{o} t\right), \\
\vec{v}_{y}^{(1)}=\left[v_{1 y}^{(1)}+v_{2 y}^{(1)} \exp i\left(k_{w} z\right)\right] E_{o} \exp i\left(k_{o} z-\omega_{o} t\right), \\
\vec{v}_{z}^{(1)}=\left[v_{1 z}^{(1)} \exp i\left(k_{w} z\right)\right] E_{o} \exp i\left(k_{w} z-\omega_{o} t\right) .
\end{gathered}
$$

where,

$$
\begin{gathered}
\Omega_{q}=v_{F}^{2}+\frac{\hbar^{2} k_{o}^{2}}{4 m^{2} \gamma_{o}}, \gamma_{o} \approx\left(1+a_{o}^{2} / 2\right)^{1 / 2} \\
a_{o}=e E_{o} / m \omega_{o} c, v_{1 x}^{(1)}=\frac{\Omega_{q} k_{o} \eta_{1 x}^{(1)}}{n_{o} \omega_{o}}+\frac{\mu_{B} S_{o} k_{o}}{m \gamma_{o} \hbar \omega_{o}}, \\
v_{2 y}^{(1)}=\left[\frac{2 S_{o} k_{\omega} B_{o w}}{i \hbar^{2} \omega_{o}^{2}}+\frac{k_{o} \Omega_{q} \eta_{2 y}^{(1)}}{n_{o} \omega_{o}}\right] \text { and } \\
v_{1 z}^{(1)}=\left[\frac{k_{o} \Omega_{q}}{n_{o} \omega_{o}^{2}}\left\{\omega_{o} \eta_{1 z}^{(1)}-i \omega_{o w} \eta_{1 x}^{(1)}\right\}-\frac{i \omega_{o w} S_{o} \mu_{B} k_{o}}{m \gamma_{o} \hbar \omega_{o}^{2}}\right] .
\end{gathered}
$$

In terms of $\gamma_{o}$ and the plasma frequency, the refractive index $\eta_{o}$ can be written as, $\eta_{o}=1-\frac{\omega_{P}^{2}}{2 \omega_{o}^{2} \gamma_{o}}$, ( in the limit of $\left.\omega_{p}^{2} / \omega_{o}^{2}<<1\right)$. From the above equations of velocities it is evident that wiggler field effects the motion of electron both in longitudinal as well as in transverse direction. Due to the above oscillations there is first order perturbation in density

$$
\begin{aligned}
& n_{x}^{(1)}=\eta_{1 x}^{(1)} E_{o} \exp i\left(k_{o} z-\omega_{o} t\right), \\
& n_{y}^{(1)}=\left[\eta_{1 y}^{(1)}+\eta_{2 y}^{(2)} \exp i\left(k_{w} z\right)\right] E_{o} \exp i\left(k_{o} z-\omega_{o} t\right), \\
& n_{z}^{(1)}=\left[\eta_{1 z}^{(1)} \exp i\left(k_{w} z\right)\right] E_{o} \exp i\left(k_{o} z-\omega_{o} t\right) .
\end{aligned}
$$

which has been calculated using continuity equation (eq. 4) with,

$$
\begin{gathered}
\xi=1-\frac{k_{o}^{2} \Omega_{q}}{\omega_{o}^{2}}, \eta_{1 x}^{(1)}=\frac{\mu_{b} S_{o} n_{o} k_{o}^{2}}{m \gamma_{o} \hbar \xi \omega_{o}^{2}}, \\
\eta_{1 y}^{(1)}=\frac{\mu_{B} S_{o} n_{o} k_{o}^{2}}{m \gamma_{o} \hbar \xi \omega_{o}^{2}}, \eta_{2 y}^{(1)}=\frac{2 S_{o} n_{o}\left(k_{o}+k_{w}\right) \cdot k_{w} B_{o w} \mu_{B}^{2}}{i \xi \hbar^{2} \omega_{o}^{3}}, \text { and } \\
\eta_{1 z}^{(1)}=\frac{i \mu_{B} S_{o} \omega_{o w}}{m \gamma_{o} \hbar \xi \omega_{o}^{3}}\left[-\frac{\Omega_{q} n_{o} k_{o}^{4}}{\xi \omega_{o}^{2}}-i k_{o}\left(k_{o}+k_{w}\right)\right] .
\end{gathered}
$$

Under the influence of the wiggler magnetic field, electron attains a spin angular moment. The dynamics of spin angular moment leads to change in dispersion. The first order perturbed spin angular momentum is obtained using the perturbative expansion of eq. (3),

$$
\begin{gathered}
\vec{S}_{x}^{(1)}=\left[S_{1 x}^{(1)} \exp i\left(k_{w} z\right)\right] E_{o} \exp i\left(k_{o} z-\omega_{o} t\right), \\
\vec{S}_{y}^{(1)}=S_{1 y}^{(1)} E_{o} \exp i\left(k_{o} z-\omega_{o} t\right) \\
\vec{S}_{z}^{(1)}=S_{1 z}^{(1)} E_{o} \exp i\left(k_{o} z-\omega_{o} t\right) .
\end{gathered}
$$

where,

$$
S_{1 x}^{(1)}=-\frac{2 S_{o} B_{o w} \mu_{B}^{2}}{\omega_{o}^{2} \hbar^{2} \gamma_{o}}, S_{1 y}^{(1)}=\frac{\mu_{B} S_{o}}{i \hbar \gamma_{o} \omega_{o}} \text {, and } S_{1 z}^{(1)}=\frac{i \mu_{B} S_{o}}{\hbar \gamma_{o} \omega_{o}} \text {. }
$$

The perturbed density and spin motion of electrons due to oscillatory velocities generate the oscillating currents. The current density is the sum of conventional source current $\left(J_{c}=-n e v\right)$ and the spin current due to spin angular magnetic moment $\left(J_{s}=-\frac{2 \mu_{B}}{\hbar} \nabla(n \times \vec{S})\right)$, whose components are,

$$
\begin{gathered}
J_{x}^{(1)}=J_{c x}^{(1)}+J_{s x}^{(1)}, \\
=\left[J_{1 x}^{(1)}+J_{2 x}^{(1)} \exp i\left(k_{w} z\right)\right] E_{o} \exp i\left(k_{o} z-\omega_{o} t\right), \\
J_{y}^{(1)}=J_{c y}^{(1)}+J_{s y}^{(1)}, \\
=\left[J_{1 y}^{(1)}+J_{2 y}^{(1)} \exp i\left(k_{w} z\right)\right] E_{o} \exp i\left(k_{o} z-\omega_{o} t\right), \\
J_{z}^{(1)}=J_{c z}^{(1)}+J_{s z}^{(1)}, \\
=\left[J_{1 z}^{(1)}+J_{2 z}^{(1)} \exp i\left(k_{w} z\right)\right] E_{o} \exp i\left(k_{o} z-\omega_{o} t\right),
\end{gathered}
$$

where,

$$
\begin{gathered}
J_{1 x}^{(1)}=-e n_{o} v_{1 x}^{(1)}-\frac{2 i k_{o} S_{o} \mu_{B} \eta_{1 x}^{(1)}}{\hbar \gamma_{o}}, \\
J_{2 x}^{(1)}=-\frac{2 i\left(k_{o}+k_{w}\right) n_{o} \mu_{B} S_{1 x}^{(1)}}{\hbar \gamma_{o}} .
\end{gathered}
$$




$$
\begin{gathered}
J_{1 y}^{(1)}=-e n_{o} v_{1 y}^{(1)}-\frac{2 i k_{o} \mu_{B}\left(n_{o} S_{1 y}^{(1)}+S_{o} \eta_{1 y}^{(1)}\right)}{\hbar \gamma_{o}}, \\
J_{2 y}^{(1)}=-e n_{o} v_{2 y}^{(1)}-\frac{2 i\left(k_{o}+k_{w}\right) S_{o} \mu_{B} \eta_{2 y}^{(1)}}{\hbar \gamma_{o}} \\
J_{1 z}^{(1)}=-\frac{2 i k_{o} \mu_{B} n_{o} S_{1 z}^{(1)}}{\hbar \gamma_{o}} \text { and } \\
J_{2 z}^{(1)}=-e n_{o} v_{1 z}^{(1)}-\frac{2 i\left(k_{o}+k_{w}\right) S_{o} \mu_{B} \eta_{1 z}^{(1)}}{\hbar \gamma_{o}} .
\end{gathered}
$$

Eqs. (15) - (17) contain the collective effects of the laser and magnetic fields on the plasma electron. The first term in eqs. (15-17) arises due to the action of the radiation field on plasma electron while the second term denotes the effect of wiggler field, under the influence of electron spin and other quantum effects.

The first order electron velocity beats with magnetic field to produce a ponderomotive force $\vec{F}_{p 2}$. The plasma electrons acquire an oscillatory velocity at $\left(2 \omega_{o}, 2 \vec{k}_{o}+\vec{k}_{w}\right)$ due to the force $F_{p 2}$, whose components are,

$$
\begin{gathered}
v_{x}^{(2)}=\left[v_{1 x}^{(2)} \exp i\left(k_{w} z\right)\right] E_{o}^{2} \exp 2 i\left(k_{o} z-\omega_{o} t\right), \\
v_{y}^{(2)}=\left[v_{1 y}^{(2)} \exp i\left(k_{w} z\right)\right] E_{o}^{2} \exp 2 i\left(k_{o} z-\omega_{o} t\right), \\
v_{z}^{(2)}=\left[v_{1 z}^{(2)}+v_{2 z}^{(2)} \exp i\left(k_{w} z\right)\right] E_{o}^{2} \exp 2 i\left(k_{o} z-\omega_{o} t\right) .
\end{gathered}
$$

where,

$$
\begin{gathered}
v_{1 x}^{(2)}=\frac{\omega_{o w}}{2 i \omega_{o}} v_{1 z}^{(2)}, v_{1 y}^{(2)}=\frac{\omega_{o w} v_{1 z}^{(1)}}{4 i \omega_{o}}+\frac{\mu_{B} k_{w} B_{o w} S_{1 y}^{(2)}}{\hbar \gamma_{o} \omega_{o}}, \\
v_{1 z}^{(2)}=-\frac{e v_{1 y}^{(1)}}{2 i \omega_{o} m \gamma_{o} c}, \text { and } v_{2 z}^{(2)}=-\frac{e v_{2 y}^{(1)}}{2 i \omega_{o} m \gamma_{o} c}
\end{gathered}
$$

The second order perturbed electron densities due to the perturbed electron velocities are,

$$
\begin{aligned}
& n_{x}^{(2)}=\left[\eta_{1 x}^{(2)}+\eta_{2 x}^{(2)} \exp i\left(k_{w} z\right)\right] E_{o}^{2} \exp 2 i\left(k_{o} z-\omega_{o} t\right), \\
& n_{y}^{(2)}=\left[\eta_{1 y}^{(2)}+\eta_{2 y}^{(2)} \exp i\left(k_{w} z\right)\right] E_{o}^{2} \exp 2 i\left(k_{o} z-\omega_{o} t\right), \\
& n_{z}^{(2)}=\left[\eta_{1 z}^{(2)}+\eta_{2 z}^{(2)} \exp i\left(k_{w} z\right)\right] E_{o}^{2} \exp 2 i\left(k_{o} z-\omega_{o} t\right) .
\end{aligned}
$$

where,

$$
\begin{gathered}
\eta_{1 x}^{(2)}=\frac{k_{o} \eta_{1 x}^{(1)} v_{1 x}^{(1)}}{\omega_{o}}, \eta_{2 x}^{(2)}=\frac{n_{o}\left(2 k_{o}+k_{w}\right) v_{1 x}^{(2)}}{2 \omega_{o}}, \\
\eta_{1 y}^{(2)}=\frac{k_{o} v_{1 y}^{(1)} \eta_{1 y}^{(1)}}{\omega_{o}},
\end{gathered}
$$

$$
\begin{gathered}
\eta_{2 y}^{(2)}=\frac{\left(2 k_{o}+k_{w}\right)}{2 \omega_{o}}\left[n_{o} v_{1 y}^{(2)}+\eta_{1 y}^{(1)} v_{2 y}^{(1)}+\eta_{2 y}^{(1)} v_{1 y}^{(1)}\right], \\
\eta_{1 z}^{(2)}=\frac{n_{o} k_{o} v_{1 z}^{(2)}}{\omega_{o}} \text { and } \eta_{2 Z}^{(2)}=\frac{n_{o}\left(2 k_{o}+k_{w}\right) v_{2 z}^{(2)}}{2 \omega_{o}}
\end{gathered}
$$

The spin angular moment is also the cause of density perturbation. The spin angular momenta of second order are,

$$
\begin{gathered}
S_{x}^{(2)}=S_{1 x}^{(2)} \exp i\left(k_{w} z\right) E_{o}^{2} \exp 2 i\left(k_{o} z-\omega_{o} t\right), \\
S_{y}^{(2)}=\left[S_{1 y}^{(2)}+S_{2 y}^{(2)} \exp i\left(k_{w} z\right)\right] E_{o}^{2} \exp 2 i\left(k_{o} z-\omega_{o} t\right), \\
S_{z}^{(2)}=\left[S_{1 z}^{(2)}+S_{2 z}^{(2)} \exp i\left(k_{w} z\right)\right] E_{o}^{2} \exp 2 i\left(k_{o} z-\omega_{o} t\right) .
\end{gathered}
$$

where,

$$
\begin{gathered}
S_{1 x}^{(2)}=\frac{\left(k_{o}+k_{w}\right) v_{1 x}^{(1)} S_{1 x}^{(1)}}{2 \omega_{o}}-\frac{\mu_{B} B_{o w} S_{1 z}^{(1)}}{i \hbar \gamma_{o} \omega_{o}}, \\
S_{1 y}^{(2)}=\frac{k_{o} v_{1 y}^{(1)} S_{1 y}^{(1)}}{2 \omega_{o}}+\frac{\mu_{B} S_{1 z}^{(1)}}{2 i \hbar \gamma_{o} \omega_{o}}, S_{2 y}^{(2)}=\frac{k_{o} v_{2 y}^{(1)} S_{2 y}^{(1)}}{2 \omega_{o}}, \\
S_{1 z}^{(2)}=-\frac{\mu_{B} S_{1 y}^{(1)}}{2 i \hbar \gamma_{o} \omega_{o}} \text { and } S_{2 z}^{(2)}=-\frac{k_{o} v_{1 z}^{(1)} S_{1 z}^{(1)}}{2 \omega_{o}}
\end{gathered}
$$

The second order electron velocity beats with the magnetic field to give rise a force $\vec{F}_{p 3}$ at $\left(3 \omega_{o}, 3 k_{o}+k_{w}\right)$. The third order electron velocity components due to this force are,

$$
\begin{aligned}
& v_{x}^{(3)}=\left[v_{1 x}^{(3)} \exp i\left(k_{w} z\right)\right] E_{o}^{3} \exp 3 i\left(k_{o} z-\omega_{o} t\right), \\
& v_{y}^{(3)}=\left[v_{1 y}^{(3)}+v_{2 y}^{(3)} \exp i\left(k_{w} z\right)\right] E_{o}^{3} \exp 3 i\left(k_{o} z-\omega_{o} t\right), \\
& v_{z}^{(3)}=\left[v_{1 z}^{(3)} \exp i\left(k_{w} z\right)\right] E_{o}^{3} \exp 3 i\left(k_{o} z-\omega_{o} t\right) .
\end{aligned}
$$

where,

$$
\begin{gathered}
v_{1 x}^{(3)}=-\frac{i \mu_{B} k_{o} S_{1 x}^{(2)}}{m \gamma_{o}}, v_{1 y}^{(3)}=\frac{e v_{1 z}^{(2)}}{2 i m \omega_{o} c \gamma_{o}}+\frac{2 \mu_{B} k_{o} S_{1 y}^{(3)}}{3 \omega_{o} m \hbar \gamma_{o}}, \\
v_{2 y}^{(3)}=\frac{e v_{2 z}^{(2)}}{2 i m \omega_{o} c \gamma_{o}}+\frac{2 \mu_{B} k_{o} S_{2 y}^{(3)}}{3 \omega_{o} m \hbar \gamma_{o}}, \text { and } v_{1 z}^{(3)}=-\frac{i e v_{1 y}^{(2)}}{3 m c \gamma_{o} \omega_{o}} .
\end{gathered}
$$

For the third-harmonic generation, the third-harmonic wave vector $k_{3}>3 k_{o}$. The phase matching condition for the process to be resonant is $\omega_{3}=3 \omega_{o}$ and $\hbar k_{3}=3 \hbar k_{o}+\hbar k_{w}$. To satisfy the phase matching condition, the required wiggler wave number $k_{w}$ is, $k_{w} \approx \frac{4}{3} \frac{\omega_{p}^{2}}{c \omega_{o} \gamma_{o}}$.

The third order perturbed density and spin magnetic moment components due to third order oscillatory velocity at $\left(3 \omega_{o}, 3 k_{o}+k_{w}\right)$ are, 


$$
\begin{gathered}
n_{x}^{(3)}=\left[\eta_{1 x}^{(3)}+\eta_{2 x}^{(3)} \exp i\left(k_{w} z\right)\right] E_{o}^{3} \exp 3 i\left(k_{o} z-\omega_{o} t\right), \\
n_{y}^{(3)}=\left[\eta_{1 y}^{(3)}+\eta_{2 y}^{(3)} \exp i\left(k_{w} z\right)\right] E_{o}^{3} \exp 3 i\left(k_{o} z-\omega_{o} t\right), \\
n_{z}^{(3)}=\left[\eta_{1 z}^{(3)} \exp i\left(k_{w} z\right)\right] E_{o}^{3} \exp 3 i\left(k_{o} z-\omega_{o} t\right) . \\
S_{x}^{(3)}=\left[S_{1 x}^{(3)} \exp i\left(k_{w} z\right)\right] E_{o}^{3} \exp 3 i\left(k_{o} z-\omega_{o} t\right), \\
S_{y}^{(3)}=\left[S_{1 y}^{(3)}+S_{2 y}^{(3)} \exp i\left(k_{w} z\right)\right] E_{o}^{3} \exp 3 i\left(k_{o} z-\omega_{o} t\right), \\
S_{z}^{(3)}=\left[S_{1 z}^{(3)}+S_{2 z}^{(3)} \exp i\left(k_{w} z\right) E_{o}^{3} \exp 3 i\left(k_{o} z-\omega_{o} t\right) .\right]
\end{gathered}
$$

where,

$$
\begin{gathered}
\eta_{1 x}^{(3)}=\frac{k_{o} \eta_{1 x}^{(2)} v_{1 x}^{(1)}}{\omega_{o}}, \\
\eta_{2 x}^{(3)}=\frac{\left(3 k_{o}+k_{w}\right)}{\omega_{o}}\left[n_{o} v_{1 x}^{(2)}+\eta_{2 x}^{(2)} v_{1 x}^{(2)}+v_{1 x}^{(2)} \eta_{1 x}^{(2)}\right], \\
\eta_{1 y}^{(3)}=\frac{k_{o}}{\omega_{o}}\left[n_{o} v_{1 y}^{(3)}+\eta_{1 y}^{(2)} v_{1 y}^{(1)}\right], \\
\eta_{1 z}^{(3)}=\frac{\left(3 k_{o}+k_{w}\right)}{3 \omega_{o}}\left[n_{o} v_{1 z}^{(3)}+\eta_{1 z}^{(1)} v_{1 z}^{(2)}\right], \\
S_{1 x}^{(3)}=\frac{\left(2 k_{o}+k_{w}\right) v_{1 x}^{(2)} S_{1 x}^{(2)}}{3 \omega_{o}}-\frac{2 \mu_{B} B_{o w} S_{1 z}^{(3)}}{3 i \omega_{o} \hbar \gamma_{o}}, \\
S_{1 y}^{(3)}=\frac{\left(2 k_{o}\right)\left(v_{1 y}^{(1)} S_{1 y}^{(2)}+v_{2 y}^{(1)} S_{1 y}^{(2)}\right)}{3 \omega_{o}}+\frac{\mu_{B} S_{1 z}^{(2)}}{3 i \omega_{o} \hbar \gamma_{o}}, \\
S_{2 y}^{(3)}=\frac{\left(2 k_{o}\right)\left(v_{1 y}^{(2)} S_{1 y}^{(1)}+v_{1 y}^{(1)} S_{2 y}^{(2)}\right)}{3 \omega_{o}}+\frac{\mu_{B} S_{2 z}^{(2)}}{3 i \omega_{o} \hbar \gamma_{o}}, \\
S_{1 z}^{(3)}=\frac{\left(k_{o}\right)\left(v_{1 z}^{(1)} S_{1 z}^{(2)}+v_{2 y}^{(1)} S_{1 y}^{(2)}\right)}{3 \omega_{o}}-\frac{\mu_{B} S_{1 y}^{(2)}}{3 i \omega_{o} \hbar \gamma_{o}} \text { and } \\
S_{2 z}^{(3)}=\frac{\left(k_{o}\right)\left(v_{2 z}^{(2)} S_{1 z}^{(1)}+v_{1 z}^{(1)} S_{1 z}^{(2)}\right)}{3 \omega_{o}}-\frac{\mu_{B} S_{2 y}^{(2)}}{3 i \omega_{o} \hbar \gamma_{o}} .
\end{gathered}
$$

The third order nonlinear source current is,

$$
\begin{gathered}
J_{x}^{(3)}=J_{c x}^{(3)}+J_{s x}^{(3)}, \\
=\left[J_{1 x}^{(3)}+J_{2 x}^{(3)} \exp i\left(k_{w} z\right)\right] E_{o}^{3} \exp 3 i\left(k_{o} z-\omega_{o} t\right), \\
J_{y}^{(3)}=J_{c y}^{(3)}+J_{s y}^{(3)}, \\
=\left[J_{1 y}^{(3)}+J_{2 y}^{(3)} \exp i\left(k_{w} z\right)\right] E_{o}^{3} \exp 3 i\left(k_{o} z-\omega_{o} t\right), \\
J_{z}^{(3)}=J_{c z}^{(3)}+J_{s z}^{(3)} .
\end{gathered}
$$

$$
=\left[J_{1 z}^{(3)}+J_{2 z}^{(3)} \exp i\left(k_{w} z\right)\right] E_{o}^{3} \exp 3 i\left(k_{o} z-\omega_{o} t\right)
$$

where,

$$
\begin{gathered}
J_{1 x}^{(3)}=-\frac{6 i k_{o} \mu_{B}}{\hbar \gamma_{o}^{2}} S_{o} \eta_{1 x}^{(3)}, J_{2 x}^{(3)}=-e n_{o} v_{1 x}^{(3)}-\left(\frac{6 i k_{o} \mu_{B}}{\hbar \gamma_{o}^{2}}\right) \\
\quad \times\left(n_{o} S_{1 x}^{(3)}+S_{o} \eta_{2 x}^{(3)}+\eta_{1 x}^{(2)} S_{1 x}^{(2)}\right), \\
J_{1 y}^{(3)}=-e n_{o} v_{1 y}^{(3)}-\left(\frac{6 i k_{o} \mu_{B}}{\hbar \gamma_{o}^{2}}\right) \times\left(n_{o} S_{1 y}^{(3)}+S_{o} \eta_{1 y}^{(3)}+\eta_{1 y}^{(2)} S_{1 y}^{(1)}\right), \\
J_{2 y}^{(3)}=-e n_{o} v_{2 y}^{(3)}-\left(\frac{2 i\left(3 k_{o}+k_{w}\right) \mu_{B}}{\hbar \gamma_{o}^{2}}\right) \times\left(n_{o} S_{2 y}^{(3)}+S_{o} \eta_{2 y}^{(3)}+\eta_{2 y}^{(2)} S_{1 y}^{(1)}\right), \\
\left.J_{1 z}^{(3)}=-\frac{6 i k_{o} \mu_{B}}{\hbar \gamma_{o}^{2}} \eta_{1 z}^{(2)} S_{1 z}^{(1)}\right), \text { and } \\
J_{2 z}^{(3)}=-e n_{o} v_{1 z}^{(3)}-\frac{2 i\left(3 k_{o}+k_{w}\right) \mu_{B}}{\hbar \gamma_{o}^{2}} \eta_{2 z}^{(2)} S_{1 z}^{(1)} .
\end{gathered}
$$

Thus, the total nonlinear current from the above equation with the oscillation of $\left(3 k_{0}+k_{w}\right)$,

$$
\begin{aligned}
& J_{3 \omega_{o}, 3 k_{o}+k_{w}}^{N L}=\left(J_{2 x}^{(3)}+J_{2 y}^{(3)}+J_{2 z}^{(3)}\right) \times \\
& E_{o}^{3} \exp i\left(\left(3 k_{o}+k_{w}\right) z-3 \omega_{o} t\right)
\end{aligned}
$$

Here the harmonic of laser field only under the influence of wiggler magnetic field have been taken into account neglecting the harmonics of the wiggler.

There also exists a self- consistent third- harmonic field

$$
\vec{E}_{3 \omega_{o}}=E_{3} \exp i\left(\left(3 k_{o}+k_{w}\right) z-3 \omega_{o} t\right) .
$$

The linear current density due to the above field is,

$$
J_{3 \omega_{o}}^{L}=-\frac{n_{o} e^{2} \vec{E}_{3}}{3 i m \omega_{o}} .
$$

\section{Third-harmonic generation}

The third harmonic field is governed by wave equation,

$$
\begin{aligned}
& \frac{\partial^{2} \vec{E}_{3 \omega_{o}}}{\partial z^{2}}-\frac{1}{c^{2}} \frac{\partial^{2} \vec{E}_{3 \omega_{o}}}{\partial t^{2}}-\frac{1}{c^{2}} \frac{\partial \vec{J}_{3 \omega_{o}}^{L}}{\partial t}=-\frac{4 \pi}{c^{2}} 3 i \omega_{o} J_{3 \omega_{o}, 3 k_{o}+k_{w}} \\
& =\zeta \exp i\left(\left(3 k_{o}+k_{w}\right) z-3 \omega_{o} t\right)
\end{aligned}
$$

where, $\zeta=\left(J_{2 x}^{(3)}+J_{2 y}^{(3)}+J_{2 z}^{(3)}\right)\left(\frac{a_{o} \omega_{o} m c}{e}\right)^{3}$ By simplifying the above equation and considering group velocity of third harmonic as $c$ and

$$
k_{3}=\left(3 \omega_{o} / c\right)\left(1-\frac{\omega_{p}^{2}}{18 \omega_{o}^{2} \gamma_{o}}\right)
$$


we get

$$
\frac{\partial E_{3}}{\partial z}+\frac{1}{c} \frac{\partial E_{3}}{\partial t}=\frac{\zeta}{2 i k_{3}}
$$

Using new set of variables $z^{\prime}=z, t^{\prime}=t-z / c$ the above equation reduces to

$$
\frac{\partial E_{3}}{\partial z}=\frac{\zeta}{2 i k_{3}} e^{-i \Delta z}
$$

Here, $\Delta=k_{3}-3 k_{o}+k_{w}$. For the Gaussian pulse

$$
\begin{gathered}
a_{o}^{2}=a_{o o}^{2} \exp \left(-t^{\prime 2} / \tau^{2}\right), a_{o o}=e E_{o o} / m c \omega_{o}, \\
\gamma_{o} \approx\left[\left(1+a_{o o}^{2} / 2\right) \exp \left(-t^{\prime 2} / \tau^{2}\right)\right]^{1 / 2}
\end{gathered}
$$

is a function of time. For a given $k_{w}$, one cannot have phase matching $(\Delta=0)$ for harmonic generation at all times. If one matches the wiggler wave number at the peak of the laser pulse $\left(t^{\prime}=0\right), k_{w} \approx \frac{4}{3} \frac{\omega_{o}}{c} \frac{\omega_{p}^{2}}{\omega^{2} \gamma_{o o}}$ where $\gamma_{o o}=\left(1+a_{o o}^{2} / 2\right)^{1 / 2}$. At all others times we have $\Delta=k_{w}\left(\frac{\gamma_{o o}}{\gamma_{o}}-1\right)$, and eq. (43) gives.

$$
E_{3}=\frac{\zeta\left(e^{-i \Delta\left(\gamma_{o o} / \gamma_{o}\right) z^{\prime \prime}}-1\right)}{2 k_{3} k_{w}\left(\gamma_{o o} / \gamma_{o}-1\right)}
$$

At distance $z=L$ we obtain the normalized third harmonic wave amplitude from eq (44).

$$
\begin{aligned}
\left|\frac{E_{3}}{E_{o}}\right|=\left[\frac{\left(J_{2 x}^{(3)}+J_{2 y}^{(3)}+J_{2 z}^{(3)}\right)}{2 k_{3} k_{w}}\left(\frac{a_{o} \omega_{o} m c}{e}\right)^{2}\right] . \\
\\
\frac{e^{-3 t^{2} / \tau^{2}}}{\gamma_{o}^{2}} \frac{\operatorname{Sink}_{w} L\left(\gamma_{o o} / \gamma_{o}-1\right)}{\left(\gamma_{o o} / \gamma_{o}-1\right)}
\end{aligned}
$$

Thus, the efficiency of third harmonic generation process is

$$
\eta_{3}=\left|\frac{E_{3}}{E_{o}}\right|^{2}
$$

In figures 1 and 2 , the variation of normalized wiggler wave number with $\left(t^{\prime} / \tau\right)$ is shown at different values of $a_{o}\left(=e E_{o} / m \omega_{o} c\right)$ has been shown for $\omega_{p} / \omega_{o}=0.2$ and 0.3 respectively. The required wave number is smaller for higher values of $a_{o}$ and larger for higher plasma density and pulse duration. The magnetic wiggler is employed to provide extra momentum for resonant third harmonic generation. However, due to short pulse duration the phase matching can be achieved for an instant only at particular wave number.

The efficiency of third harmonic generation has been plotted as a function of normalized wiggler frequency $\left(\omega_{o w} / \omega_{o}\right)$, for different values of plasma density in figure 3 . The figure shows that for a constant plasma density, the harmonic grows with an increase in the wiggler field. Maximum efficiency appears at higher densities with increase in the wiggler field. The efficiency is maximum at an instant when the phase matching is satisfied and thereafter decreases at later duration of laser pulse. The maximum efficiency is attained at about $\omega_{o w} / \omega_{o} \approx 0.087$. The cut off value for the harmonic generation and the saturation value for magnetic field also increase with plasma density. The strong magnetic field and quantum effects both contribute to increase in the cut-off value of second harmonic generation.

Figure 4 shows the variation of efficiency of third harmonic generation as a function of normalized electric field parameter $a_{o}$ at different densities. The efficiency increases with the intensity of the laser. However, for relativistic regime $\left(a_{o} \geq 1\right)$ the efficiency tends to saturate.

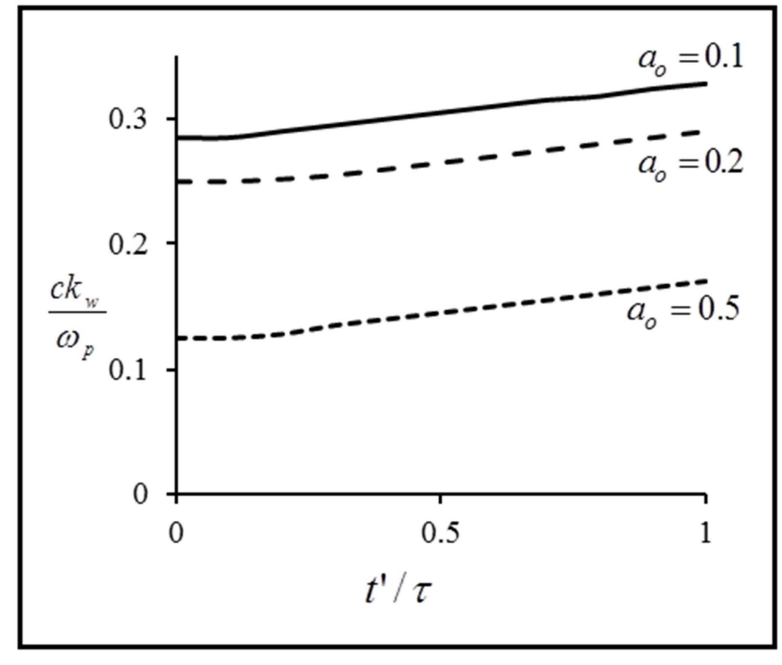

Figure 1. Variation of normalized wiggler wave number $c k_{w} / \omega_{p}$ with $t^{\prime} / \tau$ for $a_{o}=0.1,0.2$, and 0.5 at $\omega_{p} / \omega_{o}=0.1$ and $n_{o}=10^{28} \mathrm{~cm}^{-3}$.

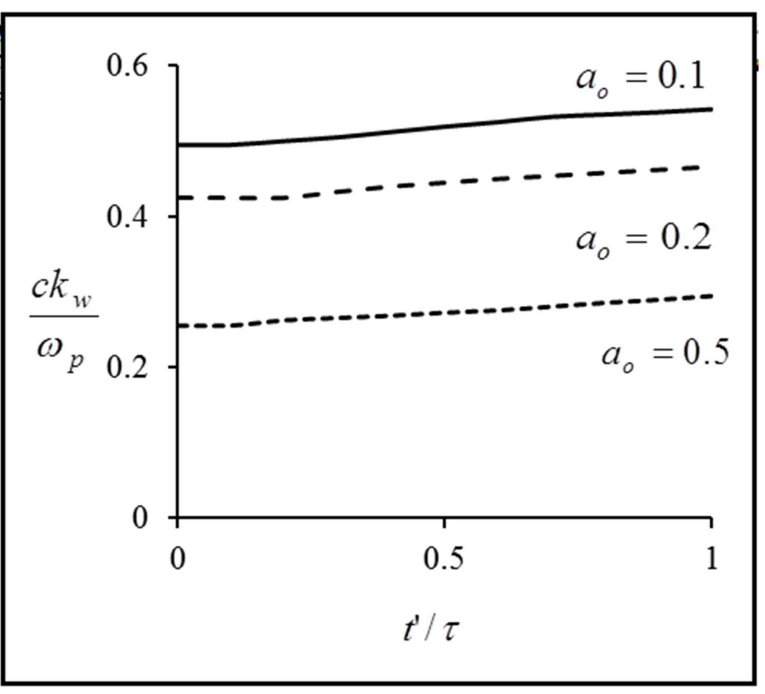

Figure 2. Variation of normalized wiggler wave number $c k_{w} / \omega_{p}$ with $t^{\prime} / \tau$ for $a_{o}=0.1,0.2$, and 0.5 at $\omega_{p} / \omega_{o}=0.2$ and $n_{o}=10^{28} \mathrm{~cm}^{-3}$. 


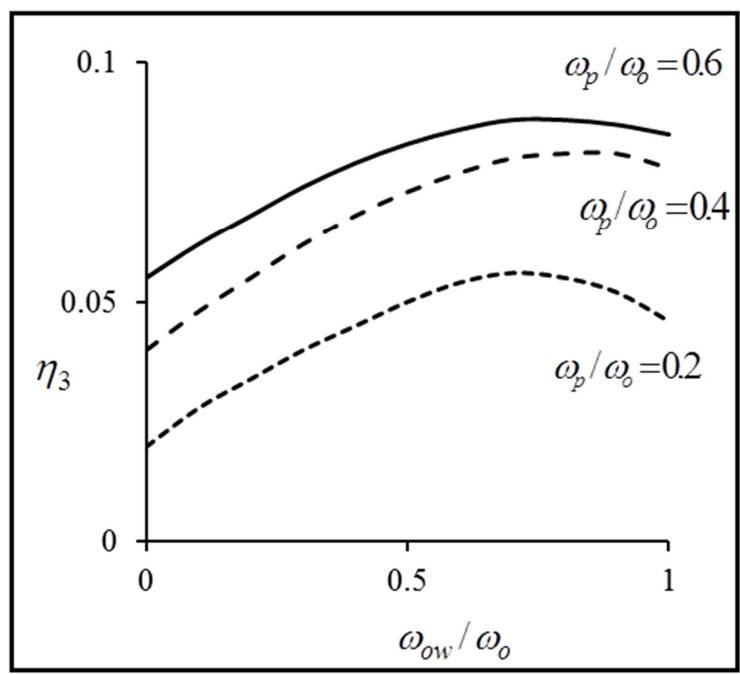

Figure 3. Variation of efficiency of phase-matched third harmonic with respect to the normalized wiggler frequency $\left(\omega_{o w} / \omega_{o}\right)$ for different values of normalized electron densit $\omega_{p} / \omega_{o}=0.2,0.4,0.6$, for $n_{o}=10^{28} \mathrm{~cm}^{3}$ and $a_{o}=0.271$.

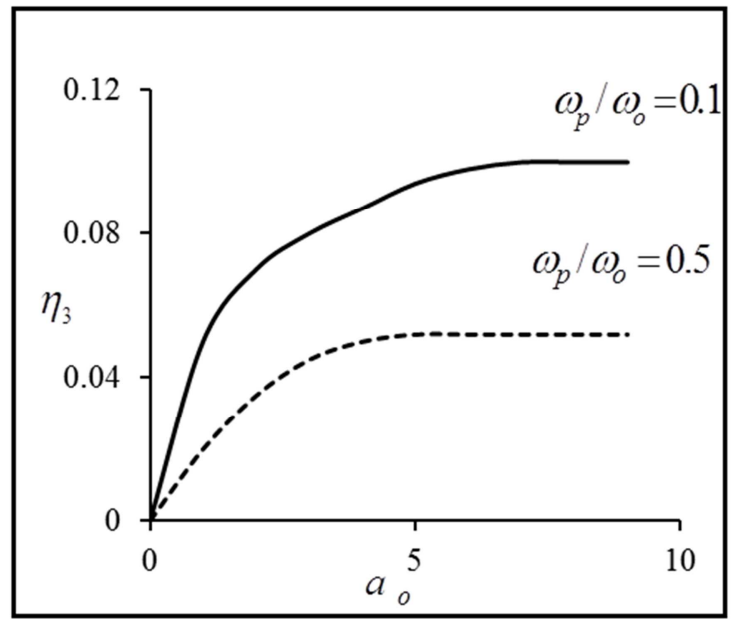

Figure 4. Variation of efficiency of phase- matched third harmonic with respect to the laser intensity $\left(a_{o}\right)$ for different values of normalized electron

density $\omega_{p} / \omega_{o}=0.1, \quad 0.5$ with $\omega_{o w} / \omega_{o}=0.2$ and $n_{o}=10^{28} \mathrm{~cm}^{3}$.

\section{Conclusion}

The power efficiency of third harmonic generation in quantum plasma for a Gaussian laser pulse using QHD model has been analysed taking into account the polarization and quantum effects. The efficiency of third harmonic generation is affected by the strength of magnetic field. The wiggler magnetic field provides the extra momentum for phase matching. The magnetic field enhances the efficiency of third harmonic generation to a significant level. Under the influence of quantum effects and magnetic field, the efficiency of generation of third harmonic is lager for phase matching condition than the phase mismatch condition. It is worth mentioning that in low-density plasma we need a super strong magnetic field to get maximum power efficiency of harmonic generation whereas in quantum plasma, which is highly dense, the excitation of efficient harmonics becomes easy by applying lesser magnetic field strength. A balance between the plasma density and applied field is required to obtain optimum efficiency.

\section{Acknowledgement}

This work was performed under the financial assistance from the UGC India, under fellowship award letter no. F.41/2006(BSR)/2007 (BSR) dated 22 ${ }^{\text {nd }}$ Oct., 2013.

\section{References}

[1] E. Esarey et al.,"Nonlinear analysis of relativistic harmonic generation by intense lasers in plasma", IEEE Trans. Plasma Sci., vol 21, pp. 95-104, 1993.

[2] W. B.Mori, C. D. Decker,andW. P. Leemans, "Relativistic harmonic content of nonlinear electromagnetic waves in underdense plasmas", IEEE Trans. Plasma Sci., vol. 21,pp. 110-119, 1993.

[3] L. M. Goldman, W.Seka, K. Tanaka, R. Short,and A. Simon, "The use of laser harmonic spectroscopy as a targetdiagnostic," Can. J. Phys., vol. 64, pp. 969-976, 1986.

[4] C Yamanaka, T. Yamanaka,T.Sasaki, J. Mizui, and H. B.Kang., "Brillouin scattering and parametric double resonance in laser produced plasmas",Phys. Rev. Lett., vol 32, pp.1038-1041, 1974.

[5] K. Tanakaet al., "Evidence of parametric instabilities in second harmonic spectra from $1054 \mathrm{~nm}$ laser produced plasmas”, Phys. Fluids., vol 27, pp. 2187-2190, 1984.

[6] D. P. Tewari, andV. K. Tripathi, "Second harmonic generation of upper hybrid radiation in a plasma", Phys. Rev. A. vol 21, pp. 1698-1703,1979.

[7] B. H- Ahn, W. W. Clark III, R. R. hurtz II, and C. D.Bates,"Second harmonic generation in $\mathrm{LiNbO}$ and $\mathrm{LiTaO}$ in the millimeter wave region",J. Appl. Phys., vol. 54, pp.1251$1255,1983$.

[8] X. U. Zhishan, et al.,"Second harmonic emission from laserplasma interactions", J. Appl. Phy., vol. 54, pp.4902$4908,1983$.

[9] R. Dragila, "Second harmonic generation at resonance absorption and modified plasma density profile", J. Appl. Phys.,vol 53, pp. 865-867, 1982.

[10] C. Grebogi, V. K. Tripathi,and H. H. Chen,"Harmonic generation of radiation in a steep density profile", Phys. Fluids, vol. 26, pp.1904-1908, 1983.

[11] C. Liao, P. Bundman, and G. I. Stegeman, "Second harmonic generation with surface guided waves in signal processing geometries", J. Appl. Phys., vol 54, pp.6813-6217, 1985.

[12] T. Weihan,et al., "The influence of laser frequency band width on the time and space resolved structures of harmonic generation”, Phys.Fluids., vol. 30, pp.1510-1514, 1986.

[13] B. Dromey, et al., "High harmonic generation in the relativistic limit”, Nat. Phys. Vol. 2,pp. 456-459, 2006. 
[14] S. Banerjee, A. R.Valenzuela, R. C. Shah RC, A. Maksimchuk, and D.Umstadter, " High harmonic generation in relativistic laser-plasma interaction”, Phys. Plasmas., vol. 9, 2393, 2002.

[15] R. P.Singh, S. L. Guptaand R. K. Thareja, "Third harmonic generation in air ambient and laser ablated carbon plasma", Phys. Plasmas., vol. 22, 123302, 2015.

[16] P.Jha, R. K. Mishra,G.Raj, andA. K.Upadhyay, "Second harmonic generation in laser magnetized-plasma interaction", Phys. Plasmas., vol. 14, 053107, 2007.

[17] M. Mori, E.Takahashi,and K. Kondo, "Image of second harmonic emission generated from ponderomotively excitedplasma density gradient", Phys.Plasmas., vol. 92, 812, 2002.

[18] N. Wadhwani, P. Kumar, and P Jha.,"Nonlinear Theory of propagation of Intense Laser Pulses in Magnetized Plasma", Phys. Plasmas, vol. 9, 263, 2002.

[19] H. Sharma, H. Jaloree, J. Parashar, "Magnetic field wiggler assisted third harmonic generation of a Guassian laser pulse in plasma", Turk J. Phys., vol. 37, pp. 368-374,2013.

[20] M. Singh, D. N. Gupta, "Effect of plasma channeling on third harmonic radiation generation", Proceeding of IPAC, THPRO064, Dresden Germany, 2014.

[21] N. K. Verma, E. Agrawal, P. Jha, "Phase matche second harmonic via laser plasma interaction", EPL, 109,15001, 2015 .

[22] M. Singh, D. N. Gupta, H. Suk, "Efficient second and third harmonic radiation generation from relativistic laser plasma interaction”, Phys. of Plasmas, vol. 22, 063303, 2015.

[23] M. Matsumoto, and K. Tanaka, "Quasi-phase-matched second-harmonic generation by backward propagating interaction”, EEE J. Quantum Electron.”Vol. 31, 700, 1995.

[24] X. Liu, D. Umstadter, E. Esarey, and A. Ting, "Nonlinear analysis of relativistic harmonic generation by intense laser in plasma”, IEEE Trans. Plasma Sci, vol. 90,21, 1993.

[25] A. Averchi, D. Faccio, R. Berlasso, M. Kolesik, J. V.Moloney, A. Couairon, and P.D. Trapani,"Linear X- wave generation by means of cross phase modulation in Kerr media", Phys. Rev. A., vol. 77, 021802R, 2008.

[26] A. H. Sheinfux, Z. Henis,M. Levin,and A. Zigler, "Plasma structures for quasiphase matched high harmonic generation,"Appl. Phys. Lett., vol. 98, 141110, 2011.
[27] S. Shibu, V. K.Tripathi,"Phase-matched third harmonic generation of laser radiation in a plasma channel: nonlocal effects", Phys. Lett. A., vol. 99, 239, 1998.

[28] H. A. Salih, V. K. Tripathi, and B. K. Pandey, "Second harmonic generation of a Gaussian laser beam in a self created magnetized plasma channel", IEEE Trans. Plasma Sci., vol. 31, 324, 2003.

[29] J. M. Rax, N. J. Fisch., "Phase-matched third harmonic generation in a plasma", IEEE Trans. Plasma Sci., vol. 21, 105,1993 .

[30] U. K. Sapaev, L. Babushkinand J. Herrman, "Quasi-phasematching for third harmonic generation in noble gases employing ultrasound”, Opt. Exp., vol. 20, 22753, 2012.

[31] N. Kant, D. N. Gupta, and S. Hyong,"Resonant thirdharmonic generation of a short-pulse laser from electron-hole plasmas", Phys. Plasmas., vol. 19, 013101, 2012.

[32] Q. Haque, S. Mahmood, and A. Mushtaq,"Nonlinear electrostatic drift waves in dense electron-positron-ion plasmas", Phys. Plasmas., vol. 15, 082315, 2008.

[33] P. K. Shukla, B. Eliasson,"Nonlinear aspects of quantum plasma physics", Phys. Usp., vol.53,51, 2010.

[34] P. K, Shukla, B.Eliasson,“Colloquium. Nonlinear collective interactions in quantum plasmas with degenerate electron fluids," Rev, Mod, Phys.vol. 83,885,2011.

[35] P. A. Andreev, L. S. Kuzmenkov, and M. I. Trukhanova, "Quantum hydrodynamics approach to the formation of waves in polarized two-dimensional systems of charged and neutral particles", Phys. Rev. B., vol. 84, 245401, 2008.

[36] F. A. Asenjo, J. Zamanian, M. Marklund, G. Brodin and P. Johansson, "Semi-relativistic effects in spin-1/2 quantum plasmas", arXiv. 1108, 4781, 2011.

[37] A. P. Mishra,"Dust ion-acoustic shocks in quantum dusty pairion plasmas,"Phys. Plasmas," vol. 16, 033702, 2009.

[38] C. L. Gardner, C. Ringhofer, "Smooth Quantum Potential for the Hydrodynamic Model", Physical Review E., vol. 53, pp. 157-167, 1996.

[39] P. K. Shukla, B. Eliasson, "Formation and dynamics of dark solitons and vortices in quantum electron plasmas", P.Phys. Rev. Lett., vol. 96, 24500, 2006. 